\title{
Synthesis of bimetallic gold-pallidum loaded on carbon as efficient catalysts for the oxidation of benzyl alcohol into benzaldehyde
}

\begin{abstract}
A series of catalysts consisting of palladium and gold nanoparticles loaded on carbon (Au$\mathrm{Pd} / \mathrm{C}$ ) were synthesized and evaluated for the liquid phase oxidation of benzyl alcohol to benzaldehyde. These catalysts were prepared via two procedures: impregnation and sol immobilization. They were characterized by using a transmission electron microscope, a thermogravimetric analyzer, a surface area analyzer, and ammonia temperature-programmed desorption. The catalysts were evaluated for the oxidation of benzyl alcohol via a heterogeneous catalyst and 35\% hydrogen peroxide as an oxidizing agent without the use of solvents. The experimental conditions and parameters, such as the effect of the preparation procedure, catalyst mass, temperature, time and the hydrogen peroxide ratio to benzyl alcohol oxidation, were optimized to obtain the optimum conversion percentage. Au-Pd/C prepared by sol immobilization showed excellent performance. A Fourier-transform infrared spectroscope was utilized to confirm the oxidation conversion of benzyl alcohol into benzaldehyde. Our result revealed the very facile and practical oxidation of benzyl alcohols with $\mathrm{H} 2 \mathrm{O} 2$ under mild conditions and produced products with a high conversion rate within a short time with excellent performance for five cycles. With the advantage of using cost-effective $\mathrm{H} 2 \mathrm{O} 2$ and the formation of water as a coproduct, the synthesized $\mathrm{Au}-\mathrm{Pd} / \mathrm{C}$ catalysts used in this work met the criteria for "green chemistry".
\end{abstract}

Keyword: Benzyl alcohol; Solvent-free oxidation; Hydrogen peroxide; Palladium and gold supported catalyst 